\title{
Hope, hype and biology: the current biomarker landscape in bladder cancer
}

\author{
Peter C. Black ${ }^{1}$ (i)
}

Received: 23 June 2019 / Accepted: 21 July 2019 / Published online: 27 July 2019

c) Springer-Verlag GmbH Germany, part of Springer Nature 2019

Biomarkers have been a focus of research in bladder cancer for decades. The search for the ideal urine marker for bladder cancer detection continues on multiple fronts, but with advances in molecularly targeted therapy and immune checkpoint therapy, the pursuit of predictive markers has also dramatically intensified. At the same time, our improved understanding of the biology of bladder cancer is opening the door to clinically useful predictive markers of response to cisplatin-based chemotherapy and radiation therapy. The combination of new therapies, enhanced molecular techniques and more rigorous investigation of bladder cancer is finally advancing the utility of biomarkers in this disease.

Exposure of urine to the bladder tumor has made the discovery of effective urine markers for cancer detection one of the true holy grails of bladder cancer research. For years, we have strived to find a marker that will augment, if not replace, cystoscopy, yet we have never seen a marker with adequate sensitivity and specificity to achieve this goal. In this issue of the Journal, Maas et al. [1] summarize some of the emerging markers that build on genomic analysis (especially DNA methylation, cell-free DNA and mRNA) of multiple markers rather than the more traditional approach of using a single protein marker (e.g., NMP22). Early results suggest that these markers, with further validation, may be able to replace some use of cystoscopy, especially in low-risk patients being evaluated for microhematuria, and patients under surveillance after resection of a low-risk tumor.

Urine markers ultimately represent one type of "liquid biopsy", although this term has come to refer primarily to analysis of markers circulating in blood. In this issue of the Journal, Rink et al. [2] highlight some of the exciting new developments in the realm of circulating tumor cells (CTC) and circulating tumor (ct)DNA in the peripheral blood.

\footnotetext{
Peter C. Black

pblack@mail.ubc.ca

1 University of British Columbia, Vancouver, Canada
}

Circulating tumor cells up to now have failed to reveal significant insights into disease biology, although they do have some value for prognostication. Circulating tumor DNA, on the other hand, is being studied with the intent of understanding the evolving molecular landscape of the cancer during different rounds of systemic therapy. Circulating tumor DNA is limited to analysis of mutations, fusions and copy number alterations, while CTC can be used to study protein and RNA changes. This means that they are complementary tests that should be exploited together to decipher mechanisms of metastasis and treatment resistance.

Beyond the blood and the urine, tissue remains the mainstay of marker discovery for bladder cancer. Fanitini et al. [3] review how genomic analysis of bladder tumors can be used to classify and risk stratify both muscle-invasive and non-muscle-invasive bladder cancer. RNA-based molecular subtypes represent one main pillar in our advanced understanding of bladder cancer, but specific gene alterations (e.g., FGFR3 mutation) and mutation signatures (e.g., APOBEC) are equally important, and are revealing opportunities for targeted therapy. Schardt et al. [4] have delved deeper into the question of whether some of these tissue markers, including also loss-of-function alterations in DNA damage repair genes, can predict response to systemic chemotherapy. These potential predictive markers are being tested in two different concepts in ongoing clinical trials. On the one hand, trials are trying to determine if patients with a DNA damage repair gene alteration can be managed with chemotherapy alone and without subsequent cystectomy. On the other hand, trials are testing whether patients without markers predictive of response to chemotherapy should forego chemotherapy and move straight to cystectomy. In either case, these markers are likely to individualize treatment delivery within the next few years.

Mitin and Choudhury [5] make the case that biomarkers could in principle similarly guide a patient towards trimodal therapy (TMT). Trimodal therapy is probably underutilized in the treatment of patients with localized MIBC. There 
appears to be a systematic bias against radiation-based therapy, and therefore only limited experience with it in most countries. It is particularly important to overcome this bias because a large proportion of patients with non-metastatic muscle-invasive bladder cancer are not receiving any treatment with curative intent. A marker that would identify patients likely to respond to radiation-based therapy, especially if it was non-prognostic in patients undergoing cystectomy, would aid in steering patients towards this modality. MRE-11, a DNA damage response-related protein, has the potential to act as such a marker, although further validation is required.

Nowhere is the global biomarker fury as intense as in the context of immune checkpoint blockade. These drugs have revolutionized the management of many cancers in a short period of time, but only a minority of patients benefit, and treatment comes at extraordinary financial cost, as well as with a modest risk of severe toxicity. Stühler et al. [6] in this issue of the Journal describe the current state of the art of biomarkers in development to predict response to immunotherapy in bladder cancer. Beyond PD-L1 immunohistochemistry, the two large phase-three trials comparing PD-1/PD-L1 blockade to second-line chemotherapy have not yet reported on correlative biomarkers, which promises to provide the field a big push forward.

With all the recent research activity, one could think that the sky is the limit for biomarker discovery in bladder cancer. Vlachostergios and Faltas [7] bring some thoughtful balance to the biomarker hype in their description of some of the key limitations in biomarker research. Methodological variability and tumor heterogeneity are two of the main obstacles to be overcome, as well as complex tumor-host interactions in the context of immunotherapy. Carefully planned prospective validation is required before clinical implementation of any candidate marker, but better understanding of the complex biology is equally important in optimizing biomarker design.

\section{References}

1. Maas M, Bedke J, Stenzl A, Todenhöfer T (2018) Can urinary biomarkers replace cystoscopy? World J Urol. https://doi. org/10.1007/s00345-018-2505-2

2. Rink M, Schwarzenbach H, Riethdorf S, Soave A (2018) The current role and future directions of circulating tumor cells and circulating tumor DNA in urothelial carcinoma of the bladder. World J Urol. https://doi.org/10.1007/s00345-018-2543-9

3. Fantini D, Meeks JJ (2018) Genomic classification and risk stratification of bladder cancer. World J Urol. https://doi.org/10.1007/ s00345-018-2558-2

4. Schardt J, Roth B, Seiler R (2018) Forty years of cisplatinbased chemotherapy in muscle-invasive bladder cancer: are we understanding how, who and when? World J Urol. https://doi. org/10.1007/s00345-018-2544-8

5. Mitin T, Choudhury A (2018) The role of biomarkers in bladder preservation management of muscle-invasive bladder cancer. World J Urol. https://doi.org/10.1007/s00345-018-2480-7

6. Stühler V, Maas JM, Bochem J, da Costa IA, Todenhöfer T, Stenzl A, Bedke J (2018) Molecular predictors of response to PD-1/ PD-L1 inhibition in urothelial cancer. World J Urol. https://doi. org/10.1007/s00345-018-2538-6

7. Vlachostergios PJ, Faltas BM (2019) The molecular limitations of biomarker research in bladder cancer. World J Urol 37(5):837848. https://doi.org/10.1007/s00345-018-2462-9

Publisher's Note Springer Nature remains neutral with regard to jurisdictional claims in published maps and institutional affiliations. 